\title{
ON AN INTEGRAL TEST OF R. W. BRINK FOR THE CONVERGENCE OF SERIES
}

\author{
BY C. T. RAJAGOPAL
}

1. Introduction. The test in question is embodied in the following theorem due to R. W. Brink.*

Let $\sum^{\infty} u_{n}$ be a series of positive terms. Also let $r(x)$ be a function such that (i) $r(n)=r_{n}=u_{n+1} / u_{n}$, (ii) $0<\lambda \leqq r(x) \leqq \mu$, (iii) $r^{\prime}(x) e x$ ists and is continuous, $\int^{\infty}\left|r^{\prime}(x)\right| d x$ is convergent. Then the convergence of the integral

$$
\int^{\infty} e^{\mathcal{S}^{x} \log r(t) d t} d x
$$

is necessary and sufficient for the convergence of the series $\sum^{\infty} u_{n}$.

It is the object of this note to show that Brink's theorem can be expressed in a more general form (Theorem 3 below) which leads at once to all the ratio tests for the convergence of series associated with Kummer's test. The ratio tests are thus welded into unity from a point of view somewhat different from that adopted by Pringsheim in his classical paper Allgemeine Theorie der Divergenz und Convergenz von Reihen mit positiven Gliedern. $\dagger$

2. Connection of Brink's Theorem with the Maclaurin-Cauchy Integral Test. The problem which confronts us in Brink's theorem is clearly that of setting up an integral $\int^{x} F(t) d t$ whose behaviour at infinity is reflected by a given series $\sum^{\infty} u_{n}$. When $\sum{ }^{\infty} u_{n}$ has all but a finite number of terms positive, the method employed to establish the Maclaurin-Cauchy integral test shows that the convergence of $\int^{\infty} F(x) d x$ is sufficient for that of $\sum^{\infty} u_{n}$ if for $n \leqq x \leqq n+1,0<u_{n} \leqq F(x),(n=m, m+1, \cdots)$. Denoting $u_{n+1} / u_{n}$ by $r_{n}$, the condition assumed is that

$$
r_{n-1} \cdot r_{n-2} \cdots r_{m} \leqq \frac{F(x)}{u_{m}}, \quad(n \leqq x \leqq n+1),
$$

* R. W. Brink, A new integral test for the convergence and divergence of infinite series, Transactions of this Society, vol. 19 (1918), p. 188.

$\dagger$ Mathematische Annalen, vol. 35 (1890), pp. 359-372. 
that is,

$$
\begin{aligned}
\sum_{\nu=m}^{n-1} \log r_{\nu} & \leqq \log \frac{F(x)}{F(m)}+\log \frac{F(m)}{u_{m}} \\
& =\int_{m}^{x} \frac{F^{\prime}(t)}{F(t)} d t+\log \frac{F(m)}{u_{m}}
\end{aligned}
$$

or,

$$
\begin{aligned}
\sum_{\nu=m}^{n-1}\left[\log r_{\nu}-\int_{\nu}^{\nu+1} \frac{F^{\prime}(t)}{F(t)} d t\right]-\int_{n}^{x} \frac{F^{\prime}(t)}{F(t)} d t \leqq & \log \frac{F(m)}{u_{m}}, \\
& (n \leqq x \leqq n+1) .
\end{aligned}
$$

The right-hand member of the above inequality may be altered to any arbitrary constant; for this would merely imply the multiplication of $F(x)$ by a positive constant in our initial hypothesis. Also, for the truth of the altered inequality the following conditions are sufficient:

(i) $\frac{F^{\prime}(x)}{F(x)}$ is bounded and integrable for $x \geqq m$,

(ii) $\log r_{\nu}-\int_{\nu}^{\nu+1} \frac{F^{\prime}(x)}{F(x)} d x \leqq \delta_{\nu}$,

where $\sum^{n} \delta_{\nu}$ is bounded above as $n \rightarrow \infty$, which is a consequence of

$$
\log r_{\nu}-\frac{F^{\prime}(x)}{F(x)} \leqq \delta_{\nu}, \quad(\nu \leqq x \leqq \nu+1) .
$$

If we put $F^{\prime}(x) / F(x)=f(x)$, the integral whose convergence is sufficient for that of $\sum^{\infty} u_{n}$ assumes the form $\int^{\infty} e^{\int^{x_{f}(t) d t}} d x$. Further, the divergence of this integral is sufficient for the divergence of $\sum^{\infty} u_{n}$ provided that in (ii) above the inequality sign is reversed and $\sum^{n} \delta_{\nu}$ is bounded below. Hence we are led to formulate the test as follows.

Theorem 1. Let $\sum^{\infty} u_{n}$ be a series of positive terms and $r_{n}=u_{n+1} / u_{n}$. If

(i) $f(x)$ is bounded and integrable for $x \geqq m$, and 
(C): $\quad \int^{\infty} e^{f^{x} f(t) d t} d x$ is convergent, $\left\{\operatorname{or}(\mathcal{D}): \quad \int^{\infty} e^{\mathcal{S}^{x} f(t) d t} d x\right.$ is divergent $\}$

(ii) for $n \leqq x \leqq n+1$,

(C): $\quad \log r_{n} \leqq f(x)+\delta_{n}, \sum^{n} \delta_{\nu}$ being bounded above, $\left\{\right.$ or $(\mathcal{D}): \quad \log r_{n} \geqq f(x)+\delta_{n}^{\prime}, \sum^{n} \delta_{\nu}^{\prime}$ being bounded below $\} ;$ then $\sum^{\infty} u_{n}$ is convergent $\{$ or divergent $\}$.

The direct proof of the theorem is exactly on the lines of that of Theorem 2 given below.

Brink's Integral Test. This is an immediate deduction from Theorem 1. For if $r(x)$ is defined as in Brink's theorem, then

and

$$
\log r(x)-\log r_{n}=\int_{n}^{x} \frac{r^{\prime}(t)}{r(t)} d t
$$

$$
\left|\log r(x)-\log r_{n}\right| \leqq \frac{1}{\lambda} \int_{n}^{n+1}\left|r^{\prime}(t)\right| d t, \quad(n \leqq x \leqq n+1) \text {. }
$$

Hence replacing $f(x)$ by $\log r(x)$ and taking

$$
\delta_{n}=\frac{1}{\lambda} \int_{n}^{n+1}\left|r^{\prime}(t)\right| d t, \quad \delta_{n}^{\prime}=-\frac{1}{\lambda} \int_{n}^{n+1}\left|r^{\prime}(t)\right| d t,
$$

we see that $\sum^{\infty} u_{n}$ converges or diverges with

$$
\int^{\infty} e^{\mathcal{S}^{x} \log r(t) d t} d x
$$

Thus Theorem 1 includes Brink's integral test, as one of his own theorems in the Annals of Mathematics* includes Hardy's generalization of the Maclaurin-Cauchy integral test $\dagger$

* R. W. Brink, $A$ new sequence of integral tests for the convergence and divergence of infinite series, Annals of Mathematics, (2), vol. 21 (1919-20), p. 41.

$\dagger \mathrm{G}$. H. Hardy, Theorems connected with Maclaurin's test for the convergence of series, Proceedings of the London Mathematical Society, (2), vol. 9 (1911). 
3. Preliminary Theorems and Deductions. Theorem 1 admits of the following generalization.

TheOREm 2. Let $\sum^{\infty} u_{n}$ be a series of positive terms and $r_{n}=u_{n+1} / u_{n}$. If

(i) $\left(D_{n}\right)$ is a strictly increasing sequence tending to infinity;

(ii) $d_{n} \equiv D_{n}-D_{n-1}=O(1)$;

(iii) $f(x)$ is bounded and integrable for $x \geqq D_{m}$, and

(C): $\quad \int^{\infty} e^{f^{x} f(t) d t} d x$ is convergent,

$\left\{\right.$ or $(\mathcal{D}): \quad \int^{\infty} e^{\mathcal{S}^{x}(t) d t} d x$ is divergent $\}$;

(iv) for $D_{n-1} \leqq x \leqq D_{n}$,

(C): $\quad \frac{1}{d_{n}} \log r_{n} \leqq f(x)+\delta_{n}, \sum^{n} \delta_{\nu} d_{\nu}$ being bounded above, $\left\{\right.$ or $(\mathcal{D}): \quad \frac{1}{d_{n}} \log r_{n} \geqq f(x)+\delta_{n}^{\prime}, \sum^{n} \delta_{\nu}^{\prime} d_{\nu}$ being bounded below $\}$; then $\sum^{\infty} u_{n} d_{n}$ is convergent $\{$ or divergent $\}$.

Proof of Case (C). Since

$$
\frac{1}{d_{\nu}} \log r_{\nu} \leqq f(t)+\delta_{\nu}, \quad\left(D_{\nu-1} \leqq t \leqq D_{\nu}\right) .
$$

by integration,

$$
\log r_{\nu} \leqq \int_{D_{\nu-1}}^{D \nu} f(t) d t+\delta_{\nu} d_{\nu}
$$

Sum for $\nu=m+1, m+2, \cdots, n-1$; then

$$
\begin{aligned}
\log \frac{u_{n}}{u_{m+1}} & \leqq \int_{D_{m}}^{D_{n-1}} f(t) d t+\sum_{\nu=m+1}^{n-1} \delta_{\nu} d_{\nu} \\
& <\int_{D_{m}}^{D_{n-1}} f(t) d t+K_{1}, \quad \text { (K } K_{1} \text { fixed) } .
\end{aligned}
$$

Also, for $D_{n-1} \leqq x \leqq D_{n}$, since $|f(t)|<M$ (fixed), $d_{n}<K$ (fixed). we have 


$$
-K M<\int_{D_{n-1}}^{x} f(t) d t .
$$

Add the last two inequalities; then

$$
\log \frac{u_{n}}{u_{m+1}}<\int_{D_{m}}^{x} f(t) d t+K_{1}+K M,\left(D_{n-1} \leqq x \leqq D_{n}\right),
$$

and

$$
u_{n}<u_{m+1} e^{K_{1}+K M} e_{\mathcal{D}_{m}}^{\int^{f}(t) d t}, \quad\left(D_{n-1} \leqq x \leqq D_{n}\right) .
$$

Hence, integrating from $D_{n-1}$ to $D_{n}$, we have

$$
u_{n} d_{n}<u_{m+1} e^{K_{1}+K M} \int_{D_{n-1}}^{D_{n}} e^{\mathcal{S}_{D_{m}}^{x} f(t) d t} d x .
$$

Compare the series $\sum^{\infty} u_{n} d_{n}$ with the series of positive terms $\sum^{\infty} \int_{D_{n-1}}^{D_{n}} e \int_{D_{m}}^{x} f(t) d t d x$, and the test for convergence (C) follows at once. The test for divergence $(D)$ is similarly proved.

The following is an adjunct to Theorem 2 .

TheOREM 2a. In Theorem 2, suppose the condition $d_{n}=O(1)$ is dropped and $f(x)<0$ (that is, the integrand in the test integral is a strictly decreasing function). If other conditions remain the same $\sum^{\infty} u_{n+1} d_{n}$ is convergent in case (C) and $\sum^{\infty} u_{n} d_{n}$ is divergent in case (D).

A slight modification is required in our former proof of $(\mathcal{C})$ :

$$
\log \frac{u_{n+1}}{u_{m+1}}<\int_{D_{m}}^{D_{n}} f(t) d t+K_{1} .
$$

Also, for $D_{n-1} \leqq x \leqq D_{n}$,

$$
0 \leqq-\int_{x}^{D_{n}} f(t) d t
$$

We add the last two inequalities, and obtain

$$
\log \frac{u_{n+1}}{u_{m+1}}<\int_{D_{m}}^{x} f(t) d t+K_{1}, \quad\left(D_{n-1} \leqq x \leqq D_{n}\right) ;
$$

whence, as before, 


$$
u_{n+1} d_{n}<u_{m+1} e^{K_{1}} \int_{D_{n-1}}^{D_{n}} e^{f_{D_{m}}^{x} f(t) d t} d x
$$

and the desired result follows by comparison.

Deductions From Theorem 2a. (i) Taking $f(x)=-\rho<0$, $\delta_{n}=0$, and putting $u_{n+1} d_{n}=a_{n}$, we see that the condition

$$
\frac{1}{d_{n}} \log \frac{a_{n} \cdot d_{n-1}}{a_{n-1} \cdot d_{n}} \leqq-\rho<0
$$

is sufficient* for the convergence of $\sum^{\infty} a_{n}$.

(ii) The condition

$$
\begin{aligned}
\frac{1}{d_{n}} \log \frac{a_{n} \cdot d_{n-1}}{a_{n-1} \cdot d_{n}} \leqq & -\frac{1}{D_{n-1}}-\frac{1}{D_{n-1} \cdot l_{1} D_{n-1}}-\cdots \\
& -\frac{\alpha}{D_{n-1} \cdot l_{1} D_{n-1} \cdots l_{p} D_{n-1}}, \quad(\alpha>1),
\end{aligned}
$$

where $l_{1} D_{n-1}=\log D_{n-1}, l_{2} D_{n-1}=\log \log D_{n-1}, \cdots \quad($ and $n \geqq m+1$ which is such that $l_{p} D_{m}>0$ ), is sufficient for the convergence of $\sum^{\infty} a_{n}$. For this implies that

$$
\begin{array}{r}
\frac{1}{d_{n}} \log \frac{u_{n+1}}{u_{n}} \leqq-\frac{1}{x}-\frac{1}{x \cdot l_{1} x}-\cdots-\frac{\alpha}{x \cdot l_{1} x \cdots l_{p} x}, \\
\left(\alpha>1 ; D_{n-1} \leqq x \leqq D_{n}\right) .
\end{array}
$$

Hence taking

$f(x)=-\frac{1}{x}-\frac{1}{x \cdot l_{1} x}-\cdots-\frac{\alpha}{x \cdot l_{1} x \cdots l_{p} x},(\alpha>1) ; \delta_{n}=0$,

we deduce the convergence of $\sum^{\infty} a_{n}$.

(iii) Similarly the condition

$$
\begin{array}{r}
\frac{1}{d_{n}} \log \frac{a_{n+1} \cdot d_{n}}{a_{n} \cdot d_{n+1}} \geqq-\frac{1}{D_{n}}-\frac{1}{D_{n} \cdot l_{1} D_{n}}-\cdots-\frac{\alpha}{D_{n} \cdot l_{1} D_{n} \cdots l_{p} D_{n}}, \\
(\alpha \leqq 1),
\end{array}
$$

is sufficient for the divergence of $\sum^{\infty} a_{n}$.

Setting $D_{n}=n$ in (ii) and (iii), we obtain Bertrand's logarithmic criteria for convergence and divergence.

\footnotetext{
* A. Pringsheim, loc. cit., p. 370.
} 


\section{Generalization of Brink's Theorem.}

THEOREM 3. Let $\sum^{\infty} a_{n}$ be a series of positive terms. If

(i) $\left(D_{n}\right)$ is a strictly increasing sequence tending to infinity;

(ii) $d_{n} \equiv D_{n}-D_{n-1}=O(1)$;

(iii) $f(x)$ has a continuous derivative $f^{\prime}(x)$ and $\int^{\infty}\left|f^{\prime}(x)\right| d x$ is convergent;

(iv)

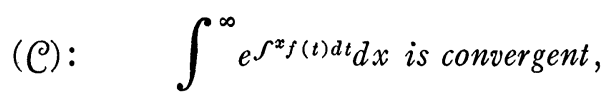

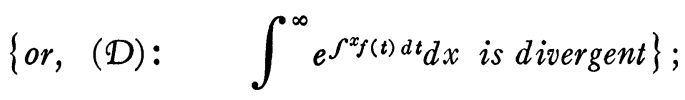

(v)

$$
\begin{aligned}
&(\mathcal{C}): \frac{1}{d_{n}} \log \frac{a_{n+1} \cdot d_{n}}{a_{n} \cdot d_{n+1}} \leqq f\left(D_{n}\right), \\
&\left\{\text { or, (D): } \quad \frac{1}{d_{n}} \log \frac{a_{n+1} \cdot d_{n}}{a_{n} \cdot d_{n+1}} \geqq f\left(D_{n}\right)\right\} ;
\end{aligned}
$$

then $\sum^{\infty} a_{n}$ is convergent $\{$ or divergent $\}$.

PRoof OF (C). Denoting $a_{n} / d_{n}$ by $u_{n}$, we have in the notation of Theorem 2,

$$
\begin{aligned}
\frac{1}{d_{n}} \log r_{n} & \leqq \int_{x}^{D_{n}} f^{\prime}(t) d t+f(x) \\
& \leqq \int_{D_{n-1}}^{D_{n}}\left|f^{\prime}(t)\right| d t+f(x), \quad\left(D_{n-1} \leqq x \leqq D_{n}\right) .
\end{aligned}
$$

Whence, choosing $\delta_{n}=\int_{D_{n-1}}^{D_{n}}\left|f^{\prime}(t)\right| d t$ in Theorem 2, we deduce the convergence of $\sum^{\infty} u_{n} d_{n} \equiv \sum^{\infty} a_{n}$.

Proof of $(\mathcal{D})$ is similar.

Deductions From Theorem 3. (i) If

$$
\frac{1}{d_{n}} \log \frac{a_{n+1} \cdot d_{n}}{a_{n} \cdot d_{n+1}}=f\left(D_{n}\right),
$$

then, under the conditions assumed, the convergence of

$$
\int^{\infty} e^{\int^{x_{f}(t) d t}} d x
$$


is necessary and sufficient for the convergence of $\sum^{\infty} a_{n}$. When $D_{n}=n$, we have Brink's theorem.

(ii) Taking $f(x)=-\rho<0$, we see that the condition

$$
\frac{1}{d_{n}} \log \frac{a_{n+1} \cdot d_{n}}{a_{n} \cdot d_{n+1}} \leqq-\rho<0
$$

is sufficient for the convergence of $\sum{ }^{\infty} a_{n} \cdot{ }^{*}$ Since $\log \gamma \leqq \gamma-1$, $(\gamma>0)$, it follows that the above condition can also be expressed in Kummer's form: $\dagger$

$$
\frac{1}{d_{n}}\left(\frac{a_{n+1} \cdot d_{n}}{a_{n} \cdot d_{n+1}}-1\right) \leqq-\rho<0 .
$$

(iii) Taking

$$
f(x)=-\frac{1}{x}-\frac{1}{x \cdot l_{1} x}-\cdots-\frac{\alpha}{x \cdot l_{1} x \cdots l_{p} x},(\alpha>1),
$$

we observe that the condition

$$
\begin{aligned}
& \frac{1}{d_{n}} \log \frac{a_{n+1} \cdot d_{n}}{a_{n} \cdot d_{n+1}} \quad \leqq-\frac{1}{D_{n}}-\frac{1}{D_{n} \cdot l_{1} D_{n}}-\cdots \\
& \text { or, } \frac{1}{d_{n}}\left(\frac{a_{n+1} \cdot d_{n}}{a_{n} \cdot d_{n+1}}-1\right) \quad-\frac{\alpha}{D_{n} \cdot l_{1} D_{n} \cdots l_{p} D_{n}}, \quad(\alpha>1) \text {, }
\end{aligned}
$$

is sufficient for the convergence of $\sum^{\infty} a_{n}$.

The corresponding divergence criterion has already been given.

Madras Christian College, TAMBARAM, SOUTH INDIA

* A. Pringsheim, loc. cit., p. 371.

$\dagger$ A. Pringsheim, loc. cit., p. 361, footnote. 